\title{
ANÁLISE DA UTILIZAÇÃO DE SALA DE CIRURGIA COM APOIO DA INFORMÁTICA
}

\author{
Maria Helena Y. Takeno Cologna * \\ Maria Eulália L. Valle Dallora ** \\ Miyeko Hayashida *** \\ Sueli Riul **** \\ Namie Okino Sawada *****
}

COLOGNA, M. H. Y. T.; DALLORA, M. E. L. V.; HAYASHIDA, M.; RIUL, S.; SAWADA, N.O. Análise da utilização de sala de cirurgia com apoio da informática. Rev.latino.am.enfermagem, Ribeirão Preto, v. 4, $\mathrm{n}^{\circ}$ especial, p. 71-81, abril 1996.

Procurou-se com este estudo verificar a possibilidade de estabelecer sistematicamente um controle de tempo do perfil das atividades desenvolvidas em salas de operação (SO) de um hospital-escola com mais de 500 leitos através da implementação de um programa informatizado, Os resultados indicam que o controle sistemático do tempo utilizado pelas equipes de enfermagem, de anestesia e de cirurgia proporcionará um aproveitamento mais racional e eqüitativo das potencialidades de recursos humanos e recursos materiais existentes.

UNITERMOS: enfermagem, informática, sala de operação

\footnotetext{
* Enfermeira, Diretor do Serviço de Centro Cirúrgico do Hospital das Clínicas da Faculdade de Medicina de Ribeirão Preto da Universidade de São Paulo - bolsista de aperfeiçoamento em pesquisa do CNPq, ligada ao projeto "A centralidade da comunicação no processo de enfermagem"

** Estatística, Assistente de Planejamento e Controle do Hospital das Clínicas da Faculdade de Medicina de Ribeirão Preto da Universidade de São Paulo

*** Enfermeira, Chefe da Seção de Apoio Laboratorial da Escola de Enfermagem de Ribeirão Preto da Universidade de São Paulo

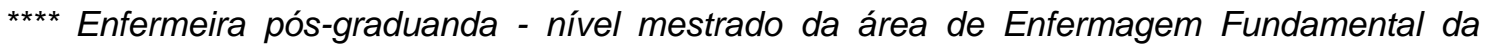
Escola de Enfermagem de Ribeirão Preto da Universidade de São Paulo

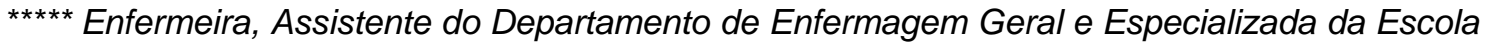
de Enfermagem de Ribeirão Preto da Universidade de São Paulo
} 


\section{INTRODUÇÃO}

O hospital é uma organização complexa, um sistema social aberto, com múltiplas finalidades, sendo a principal delas o atendimento ao cliente, a comunidade.

Há quem afirme que a eficiência de um hospital pode ser avaliada pelo funcionamento de um centro cirúrgico ${ }^{7}$.

Dos primórdios do centro cirúrgico, com registros no século XII, a evolução foi franca, progredindo até atingir a atual complexidade da Unidade de Centro Cirúrgico ${ }^{6}$.

Foram atos criativos do homem que arrancaram das velhas tradições levando-o a descobertas, criações que resultaram em grandes saltos na história, desde o domínio do fogo até a informática ${ }^{5}$. Uma conquista definitiva (e irreversível), pois a presença do computador nos mais variados setores das atividades cotidianas é uma realidade e acredita-se, já não ser mais possível retroceder nesse processo de informatização da sociedade ${ }^{2}$.

A aplicação da informática nos setores da saúde encontra-se em acelerado crescimento na sociedade brasileira ${ }^{8}$ e assim, o enfermeiro de centro cirúrgico, como responsável não só pela área assistencial, mas também pelas áreas técnicoadministrativas, tem se deparado com transformações onde os computadores começam a tornar uma constante a se relacionar com a sua prática ${ }^{4}$.

Os sistemas de computação instalados em unidades hospitalares são geralmente denominados sistemas de informação, isto porque, sua função é auxiliar na transmissão de informações relacionadas ao paciente. Os sistemas de informação computadorizada são utilizados na coleta, organização e transmissão de dados entre diversos departamentos, entre profissionais de saúde e para transmitir dados aos próprios pacientes. Uma vez que os enfermeiros desempenham um papel central no processo de comunicação relacionado ao paciente, tais sistemas oferecem à enfermagem uma extraordinária ferramenta na prestação de cuidados de melhor qualidade ao paciente ${ }^{1}$.

Espera-se o envolvimento do enfermeiro nos projetos de implementação de sistemas de dados informatizados para utilização desta tecnologia como instrumento de trabalho na otimização da prática de enfermagem ${ }^{8}$. A informatização libera o enfermeiro de tarefas rotineiras e amplia sua capacidade de reflexão e criatividade ${ }^{2,3}$.

A aplicação da informática na área de enfermagem abrange várias funções administrativas, pode-se, por exemplo, estabelecer o controle diário das salas de operação dentro de uma Unidade de Centro Cirúrgico ${ }^{3}$. Assim, em consonância com a tendência atual de implementação da informática nos diversos serviços de um hospital-escola, buscou-se verificar a viabilidade de um sistema que, através dos recursos materiais e humanos disponíveis, permita o controle de determinadas atividades desenvolvidas em sala de operação (SO). 
Este estudo foi realizado em um hospital-escola, do interior paulista, com mais de 500 leitos, cuja Unidade de Centro Cirúrgico é composta por 16 salas de operação, com horários distribuídos entre 14 especialidades cirúrgicas. Do total de salas, 06 encontram-se inativadas por insuficiência de recursos humanos e materiais, mas freqüentemente as equipes cirúrgicas solicitam aumento dos horários disponíveis.

Frente a possibilidade de atendimento a esta solicitação, tomou-se necessário um estudo sobre a demanda de tempo utilizado pelas equipes para verificar quais delas teriam real necessidade de aumentar os seus períodos.

Observa-se na prática, que algumas equipes pleiteiam insistentemente 0 aumento de horário disponível e aparentemente não ocupam sequer a cota a elas atribuídas. Não obstante, nota-se também que ocorrem atrasos entre as operações realizadas por uma determinada equipe em uma mesma sala. As equipes cirúrgicas geralmente relacionam estes atrasos a demora na limpeza de salas, entretanto, supõe-se que elas podem estar também relacionadas as equipes de anestesia, de cirurgia e ao próprio paciente.

Desta forma, com o objetivo de obter subsídios que viabilizem a distribuição eqüitativa do aumento de horários entre as especialidades cirúrgicas, utilizando o computador como uma ferramenta disponível na instituição, este estudo busca através de um sistema informatizado, verificar:

- o perfil das atividades desenvolvidas pelas equipes profissionais no centro cirúrgico, relativo ao movimento diário de cirurgias;

- o tempo disponível e o tempo efetivamente utilizados pelas equipes cirúrgicas, dentro das salas de operação;

- a ocorrência de atraso no início ou entre as cirurgias realizadas na mesma sala e pela mesma especialidade.

\section{METODOLOGIA}

Para obtenção dos dados deste estudo utilizou-se das anotações de enfermagem, constantes no impresso de notas de sala de operação (SO), preenchidas rotineiramente, pelo circulante de sala (atendente de enfermagem, auxiliar de enfermagem, técnico de enfermagem ou enfermeiro) com registro das horas de entrada e saída do paciente em SO e do início e término da anestesia e cirurgia.

Foram pesquisadas 460 notas de sala de operação de pacientes que submeteram-se ao procedimento no centro cirúrgico do Hospital em estudo, correspondentes ao mês de agosto de 1990, das especialidades cirúrgicas assim denominadas: TCV (Torácica e Cardiovascular); GAC (Gastro Cirurgia); CPL 
(Plástica); URO (Urologia); VAS (Vascular); ENP (Cabeça e Pescoço); CGT (Geral e Torácica); NEC (Neurocirurgia); CPE (Pediátrica); OFT (Oftalmologia); ORT (Ortopedia); ORL (Otorrinolaringologia); PRO (Proctologia) e GO (Ginecologia e Obstetrícia).

O total de horas disponíveis para cada especialidade foram obtidas considerando-se os períodos atribuídos conforme normas atuais de funcionamento da Unidade de Centro Cirúrgico. Desta forma, computou-se 04:30 horas para cada período da manhã e 04:30 horas para cada período da tarde.

Considerou-se tempo de anestesia o período compreendido entre o início da execução de qualquer procedimento relativo ao ato anestésico pelo anestesiologista, junto ao paciente até a liberação do mesmo, da sala cirúrgica; e tempo de cirurgia compreendido entre a diérese e a síntese.

Uma vez coletados, os dados foram registrados em planilha eletrônica, criado especificamente para este fim pelo Grupo de Avaliação de Desempenho do Hospital, através do software Lotus e utilizado um PC-A T, disponível na época.

\section{RESULTADOS E DISCUSSÃO}

Foram realizadas 460 cirurgias distribuídas entre as 14 especialidades, no período correspondente à coleta de dados. Houve uma maior freqüência de cirurgias da G. O. (18,26\%), OFT (15,22\%), ORT (13,26\%) e ORL $(9,78 \%)$, como demonstra a tabela I observando uma menor freqüência nas especialidades de CPE e CPL, cada uma correspondendo igualmente a 2,39\% das cirurgias realizadas.

Quanto ao tempo médio utilizado por cirurgia, as especialidades que demandaram maior tempo foram a NEC com uma média de 6,20 horas/cirurgia; TCV com 5,53 horas/cirurgia; ENP com 4,58 horas/cirurgia e GAC com 4,07 horas/cirurgia. O menor tempo médio utilizado por cirurgia foi a CPE com 2,04 horas/cirurgia, seguida pela OFT com tempo médio de 2,20 horas/cirurgia (Tabela I).

No tocante ao tempo que cada especialidade dispõe para operar quando confrontado com aquele efetivamente utilizado, pode-se observar na Figura I, que algumas ultrapassavam o limite previamente estipulado enquanto em outras o tempo foi subutilizado ou utilizado na sua potencialidade.

Ainda na tabela I, pode-se notar que duas especialidades, URO $(100,04 \%)$ e GAC (99,27\%), utilizaram totalmente o tempo disponível. Por outro lado, quatro especialidades extrapolaram o tempo disponível: NEC (132,65\%), VAS (121,04\%), ENP $(118,01 \%)$ e ORL $(106,55 \%)$. As demais especialidades subutilizaram o tempo disponível com destaque para CPL e CPE que aproveitaram apenas a metade do período atribuído.

Chamou-nos a atenção (Tabela I) que a especialidade CPE realizou o menor número de cirurgias $(2,39 \%)$ utilizando a menor tempo médio por cirurgia 
(2,04 horas/cirúrgia). Em contrapartida, dispõe do menor tempo disponível e ainda assim foi a especialidade que obteve o menor índice de aproveitamento deste tempo $(50 \%)$.

O tempo em que a SO permanece vaga (sem paciente), entre uma e outra cirurgia, foi em média de I (uma) hora em três especialidades: URO (1,09h), GAC $(1,05 \mathrm{~h})$ e TCV (1,00h). O menor tempo médio encontrado e único abaixo de meia hora, foi a especialidade ENP com 25 minutos em média de ociosidade da SO. (Tabela 2).

Em se tratando do tempo médio de sala vaga entre o início da primeira cirurgia escalada e o horário padronizado (previsto para às 07:30 horas), o atraso foi maior nas especialidades de CPE (49 minutos), seguida pelo ORO e TCV (ambos com 46 minutos), GAC (41 minutos) e VAS (38 minutos). A GO foi a especialidade que apresentou o menor tempo para iniciar a primeira cirurgia programada, do período da manhã, em relação ao horário previsto, com atraso médio de 16 minutos (Tabela 2).

Ainda, na Tabela 2, pode-se verificar o tempo médio consumido pela equipe de anestesia entre a chegada do paciente à SO e o início propriamente da anestesia. A equipe demorou maior tempo médio para iniciar o procedimento nos pacientes da especialidade TCV (29 minutos) decrescendo para outras especialidades como NEC (18 minutos), ORT, ENPe URO (com 16minutos igualmente para cada uma). Mais uma vez o menor tempo médio observado foi na especialidade de CPE (6 minutos) imediatamente seguida pela CGT (8 minutos).

O tempo médio observado entre o início da anestesia e início da operação foi maior para as especialidades de NEC (59 minutos) e TCV (50 minutos). Em posição intermediária encontram-se as especialidades de ORT e ENP que consumiram igualmente 31 minutos, ficando a CGT (17 minutos) e a GO e ORL (19 minutos igualmente para ambos) com os menores tempos médios gastos para começar a operação depois de iniciada a anestesia (Tabela 2).

\section{CONCLUSÕES}

Com base nos resultados encontrados neste estudo, pode-se concluir que:

- O perfil de atividades desenvolvidas pelas equipes de enfermagem, de anestesia e de cirurgia no centro cirúrgico, mostra que elas não trabalham em sincronia, o que pode ser evidenciado pelos atrasos verificados, no início das primeiras cirurgias do dia e pelo período compreendido entre o encaminhamento do paciente para o Centro de Recuperação e a entrada do próximo, da mesma especialidade na mesma SO.

- Ocorre perda de tempo entre uma e outra cirurgia de uma determinada especialidade realizada na mesma SO. Pode-se inferir que os atrasos ocorram por problemas inerentes às equipes bem como ao próprio paciente. 
- Todas as especialidades apresentaram, em maior ou menor grau, atrasos para iniciarem a primeira cirurgia, embora tenha sido observado que as saias e os pacientes estivessem preparados às 07:30 horas, conforme programado.

- As especialidades que fizeram melhor aproveitamento do tempo disponível foram: NEC, VAS, ENP, ORL, URO e GAC. Portanto, caberiam a elas a distribuição dos horários a serem ampliados.

\section{SUGESTÕES}

A utilização da informática viabilizou a organização do volume de informações deste estudo, tendo como vantagem a rapidez e confiabilidade de seus cálculos. Acresça-se a isto que os recursos humanos e materiais utilizados foram disponíveis na época na Instituição. Desta forma, sugere-se maior empenho no investimento da informatização em áreas como da Unidade de Centro Cirúrgico, a exemplo de outras Instituições que já criaram condições para viabilizar o uso do computador em áreas semelhantes.

A implementação de um programa de controle informatizado no Centro Cirúrgico possibilitaria uma avaliação sistematizada das atividades desenvolvidas e conseqüentemente 0 aproveitamento racional das potencialidades de recursos humanos e materiais existentes.

Em consonância com ATKINSON et al $(1989)^{1}$, acreditamos que os sistemas de computação além de facilitar a comunicação e ajudar os enfermeiros no planejamento de cuidados ao paciente, possibilita a atualização de prescrições médicas, materiais e outros. A informação registrada no sistema pode ser partilhada simultaneamente por vários usuários e obtida de acordo com o interesse específico, reduzindo assim, o volume de tempo burocrático despendido na comunicação entre as equipes.

Propusemos com este trabalho, quantificar o tempo consumido pelas ocorrências de atrasos e ociosidade da SO. Entretanto, para análise qualitativa das causas atribuíveis, sugere-se um estudo específico mais apurado e detalhado do problema.

\section{ANALYSIS OF THE OPERATING ROOM UTILIZATION WITH THE SUPPORT OF INFORMATICS}

With this study intended to verify the possibility of settling a time control of the activities developed at the operating room of an University Hospital, which has more than 
500 beds, through the introduction of a computer program. The results indicate that this kind of systematic time control, which is used by nurses, anesthesia and surgery staff is able to offer a more rational utility to human and material resources.

UNITERMS: nursing, informatics, operating-room

\section{ANÁLISIS DE LA UTILIZACIÓN DE LA SALA DE CIRUGÍA CON APOYO DE LA INFORMÁTICA}

Con este estudio se buscó verificar la posibilidad de se establecer sistemáticamente un control del tiempo, sobre el perfil de las actividades desarrolladas en salas quirúrgicas de un hospital-escuela con más de 500 lechos, Los resultados indican que el control sistemático del tiempo utilizado por los equipos de enfermería, de anestesia y de cirugía proporcionará un provecho más racional y equitativo de las potencialidades de recursos humanos y recursos materiales existentes.

UNITERMOS: enfermería, informática, quirófano

\section{REFERÊNCIAS BIBLIOGRÁFICAS}

01. ATKINSON, L. D.; MURRA Y. M. E. Sistemas de informação computadorizados instrumentos de comunicação para a enfermagem. In: Fundamentos de enfermagem: introdução ao processo de enfermagem. Rio de Janeiro: Guanabara, 1989. cap. 10, p. 113-41.

02. DEMENEGHI, L. S. M.; SCHIMITT, M. T. Implantação da informatização em área cirúrgica. Fase primeira. Rev.Paul.Enfermagem, São Paulo, p. 54-6, jul. 1991. Ed. especial.

03. ÉVORA, Y. D. M.; SCOCHI, C. G. S.; SANTOS, B. R. L. O computador como instrumento de apoio na assistência e administração de enfermagem. Rev.Gaúch.Enfermagem, Porto Alegre, v. 12, n. 1, p, 41-5, jan. 1991.

04. LUIS, M. V.; SCOCHI, C. G. S.; VON ATZINGEN, R. H. Reflexões sobre a inserção da informática na profissão de enfermagem. Rev.Gaúch.Enfermagem, Porto Alegre, v. 13, n. 1, p. 37-40, jan. 1992.

05. MARZIALE, M. H. P.; ZANETTI, M. L.; CASSIANI, S. H. D. B. Tecnologia em enfermagem no centro cirúrgico: análise de publicações nacionais. In: JORNADA DE ENFERMAGEM EM CENTRO CIRÚRGICO DO ESTADO DE SÃO PAULO, 3., Ribeirão Preto, jul. 1989. p. 166-75. 
06. RIBEIRO, F.H. Regulamento do centro cirúrgico de um hospital de 500 leitos. Rev.Paul.Enfermagem, São Paulo, v.17, n. 7, p. 38-60, jul. 1969.

07. SALZANO, S.D.T. A enfermeira de centro cirúrgico frente ao preparo e utilização do instrumental cirúrgico, com destaque para lâminas e cabo de bisturi. Rev.Paul.Enfermagem, São Paulo, v. 4, n. 1, p. 20-6, jan. /mar. 1984.

08. SCOCHI, C.G.S.; SANTOS, B.R.L.; ÉVORA, Y.D.M. A informática na prática de enfermagem: um novo desafio para o enfermeiro. Rev.Gaúch.Enfermagem, Porto Alegre, v. 12, n. 2, p. 19-22, jul. 1991. 
TABELA 1 - DISTRIBUIÇÃO NUMÉRICA DAS CIRURGIAS REALIZADAS, DO TEMPO MÉDIO UTILIZADO POR CIRURGIA, DO TEMPO DISPONÍVEL E UTILIZADO E PERCENTUAL POR ESPECIALIDADE CIRÚRGICA (AGOSTO, 1990)

\begin{tabular}{l|cccccc}
\hline \multirow{2}{*}{$\begin{array}{l}\text { ESPECIALIDADES } \\
\text { CIRÚRGICAS }\end{array}$} & $\begin{array}{c}\text { CIRURGIAS } \\
\text { REALIZADAS }\end{array}$ & $\begin{array}{c}\text { TEMPO } \\
\text { MÉDIO POR } \\
\text { CIRURGIA }\end{array}$ & $\begin{array}{c}\text { TOTAL } \\
\text { TEMPO } \\
\text { DISPONÍVEL }\end{array}$ & $\begin{array}{c}\text { TOTAL } \\
\text { TEMPO } \\
\text { UTILIZADO }\end{array}$ & $\begin{array}{c}\text { UTILIZAÇÃO } \\
\text { DO TEMPO } \\
\text { DISPONÍVEL }\end{array}$ \\
\cline { 2 - 7 } & No & $\%$ & Em horas & Em horas & Em horas & $\%$ \\
\hline $\begin{array}{l}\text { Torácica e } \\
\text { Cardiovascular }\end{array}$ & 17 & 3,70 & $05: 53$ & $139: 30$ & $100: 15$ & 71,89 \\
Gastrocirurgia & 25 & 5,43 & $04: 07$ & $103: 30$ & $102: 55$ & 99,27 \\
Plástica & 11 & 2,39 & $03: 02$ & $63: 00$ & $33: 25$ & 52,77 \\
Urologia & 29 & 6,30 & $03: 34$ & $103: 30$ & $103: 35$ & 100,04 \\
Vascular & 28 & 6,09 & $03: 41$ & $85: 30$ & $103: 25$ & 121,04 \\
Cabeça e PescoçO & 15 & 3,26 & $04: 58$ & $63: 00$ & $74: 35$ & 118,01 \\
Geral e Torácica & 24 & 5,22 & $02: 57$ & $85: 30$ & $71: 02$ & 83,25 \\
Neurocirurgia & 17 & 3,70 & $06: 20$ & $81: 00$ & $107: 45$ & 132,65 \\
Pediátrica & 11 & 2,39 & $02: 04$ & $45: 00$ & $22: 50$ & 50,00 \\
Oftalmologia & 70 & 15,22 & $02: 20$ & $207: 00$ & $163: 37$ & 78,92 \\
Ortopedia & 61 & 13,26 & $03: 05$ & $198: 00$ & $188: 05$ & 94,97 \\
Otorrinolaringologia & 45 & 9,78 & $02: 52$ & $121: 30$ & $129: 25$ & 106,55 \\
Proctologia & 23 & 5,00 & $02: 54$ & $81: 00$ & $66: 45$ & 82,03 \\
Ginecologia e & 84 & 18,26 & $02: 59$ & $297: 00$ & $250: 55$ & 84,36 \\
Obstetrícia & & & & & & \\
\hline
\end{tabular}


TABELA 2 - DISTRIBUIÇÃO DOS TEMPOS MÉDIOS DE OCIOSIDADE DA SO, DO ATRASO PARA INÍCIO DA $1^{\text {a }}$ CIRURGIA ENTRE A CHEGADA DO PACIENTE E INÍCIO DA ANESTESIA E ENTRE ANESTESIA E INÍCIO DA CIRURGIA POR ESPECIALIDADE CIRÚRGICA (AGOSTO, 1990)

\begin{tabular}{|c|c|c|c|c|}
\hline \multirow[b]{2}{*}{$\begin{array}{l}\text { ESPECIALIDADES } \\
\text { CIRÚRGICAS }\end{array}$} & \multicolumn{4}{|c|}{ TEMPO MÉDIO EM HORAS } \\
\hline & $\begin{array}{l}\text { Ociosidade } \\
\text { da SO }\end{array}$ & $\begin{array}{c}\text { Atraso para } \\
\text { início da } 1^{\mathrm{a}} \\
\text { cirurgia }\end{array}$ & $\begin{array}{l}\text { Entre a chegada } \\
\text { paciente e início } \\
\text { anestesia }\end{array}$ & $\begin{array}{c}\text { Entre } \\
\text { anestesia e } \\
\text { início cirurgia }\end{array}$ \\
\hline $\begin{array}{l}\text { Torácica e } \\
\text { Cardiovascular }\end{array}$ & 01:00 & $: 46$ & :29 & $: 50$ \\
\hline Gastrocirurgia & 01:05 & $: 41$ & :15 & :21 \\
\hline Plástica & $: 35$ & $: 18$ & $: 12$ & :23 \\
\hline Urologia & 01:09 & $: 46$ & :16 & :23 \\
\hline Vascular & $: 44$ & $: 38$ & :15 & :23 \\
\hline Cabeça e Pescoço & $: 25$ & $: 22$ & :16 & :31 \\
\hline Geral e Torácica & :38 & :20 & $: 08$ & $: 17$ \\
\hline Neurocirurgia & :35 & $: 25$ & :18 & $: 59$ \\
\hline Pediátrica & :35 & $: 49$ & $: 06$ & $: 25$ \\
\hline Oftalmologia & $: 40$ & $: 31$ & :12 & $: 21$ \\
\hline Ortopedia & $: 43$ & $: 29$ & :16 & $: 31$ \\
\hline Otorrinolaringologia & $: 42$ & $: 20$ & $: 10$ & :19 \\
\hline Proctologia & $: 44$ & $: 33$ & $: 13$ & $: 21$ \\
\hline $\begin{array}{l}\text { Ginecologia e } \\
\text { Obstetrícia }\end{array}$ & $: 43$ & $: 16$ & $: 14$ & :19 \\
\hline
\end{tabular}


FIGURA 1 - TEMPO DISPONÍVEL E TEMPO EFETIVAMENTE UTILIZADO PELAS EQUIPES CIRÚRGICAS, EM HORAS, DE ACORDO COM AS ESPECIALIDADES

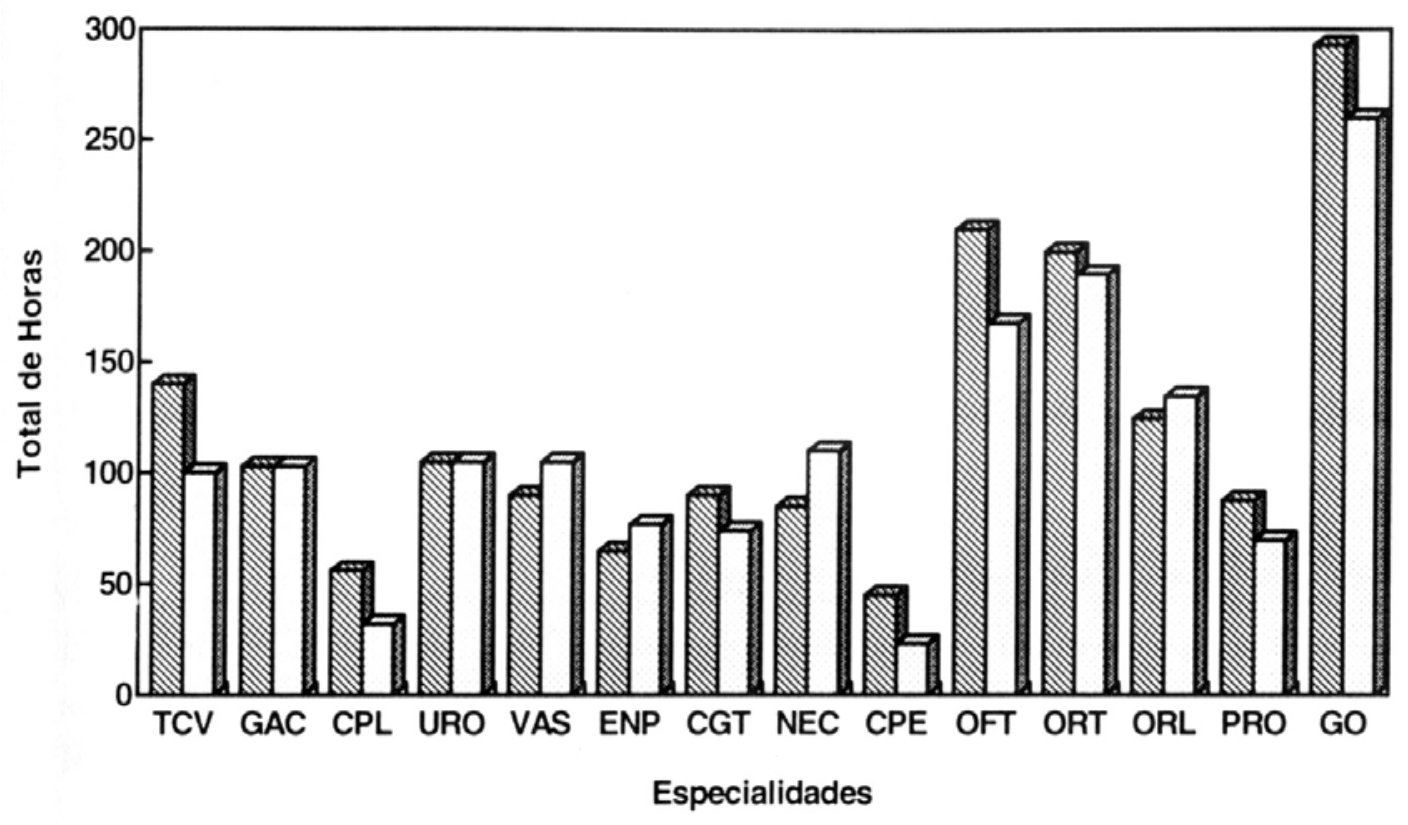

\$ Tempo Disponível 口Tempo Utilizado 